\section{Stimulus Generalization}

Joel W. Hughes

Department of Psychology, Kent State University, Kent, OH, USA

\section{Synonyms}

Generalization

\section{Definition}

Stimulus generalization is the tendency of a new stimulus to evoke responses or behaviors similar to those elicited by another stimulus. For example,
Ivan Pavlov conditioned dogs to salivate using the sound of a bell and food powder. The unconditioned stimulus (food powder) was paired with a conditioned stimulus (sound of a bell) until the conditioned stimulus produced the response (salivation) in the absence of the unconditioned stimulus (food powder). The dogs were then noted to salivate in response to other noises similar to the bell used in conditioning, even though these noises had never been used during conditioning.

\section{Cross-References}

- Stimulus Control

- Stimulus-Bound Behavior 